\title{
COMUNICAÇÃO
}

\section{COMPOSIÇÃOQUÍMICA DO MOSTO DA UVA 'CABERNET FRANC' CONDUZIDA NO SISTEMA LIRA ABERTA}

\author{
Chemical composition of the must of Cabernet Franc grape conducted in the lyre system
}

\author{
Luciano Manfroi ${ }^{1}$, Alberto Miele ${ }^{2}$, Luiz A. Rizzon ${ }^{2}$, Carlos I. N. Barradas ${ }^{3}$
}

\begin{abstract}
RESUMO
Com o objetivo de avaliar a composição físico-química do mosto da uva 'Cabernet Franc' conduzida no sistema lira aberta, foi realizado o presente trabalho em oito vinhedos durante o ciclo vegetativo de 1995/1996 nos municípios de Bento Gonçalves e Monte Belo do Sul, RS. Os valores médios das variáveis avaliadas foram similares aos obtidos na região com outros sistemas de condução, ou seja: ${ }^{\circ}$ Brix - 17,0; acidez total - 113 meq $\mathrm{L}^{-1}$; $\mathrm{pH}$ - 3,17; nitrogênio total - 246,1 $\mathrm{mg} \mathrm{L}^{-1}$; polifenóis totais (I 280) - 11,8; prolina 182,2 mg L-1; ácido tartárico - 88 meq L ${ }^{-1}$; ácido málico - 56 meq L ${ }^{-1}$; -951 mg L-1; P - 71,3 mg L-1; Ca - 34,4 mg L-1; e Mg - 50,2 mg L'. Esses resultados mostram que o sistema de condução da videira em lira aberta pode ser recomendado para a produção de uva 'Cabernet Franc' nas condições de solo e de clima da Serra Gaúcha.
\end{abstract}

Termos para indexação: Viticultura, uva, mosto, composição química.

\begin{abstract}
The present work was carried out in order to evaluate the physicochemical composition of the must of cv. Cabernet Franc. It was carried out during the 1995/1996 vegetative cycle, in eight vineyards conducted in the lyre system and located in the communes of Bento Gonçalves and Monte Belo do Sul, Rio Grande do Sul-State, Brazil. The average values of the variables showed similar results to those previously determined in the same region with other trellising systems: ${ }^{\circ}$ Brix -17.0 ; total acidity - 113 meq $\mathrm{L}^{-1}$; $\mathrm{pH}-3.17$; total nitrogen - $246.1 \mathrm{mg} \mathrm{L}^{-1}$; total polyphenols (I 280) - 11.8; proline - $182.2 \mathrm{mg} \mathrm{L}^{-1}$; tartaric acid - $88 \mathrm{meq} \mathrm{L}^{-1}$; malic acid - 56 meq L-1 $\mathrm{K}_{-}$ $951 \mathrm{mg} \mathrm{L}^{-1} ; \mathrm{P}-71.3 \mathrm{mg} \mathrm{L}^{-1}$; Ca - $34.4 \mathrm{mg} \mathrm{L}^{-1}$; and $\mathrm{Mg}-50.2 \mathrm{mg} \mathrm{L}^{-1}$. These results show that grapevines trellised in the lyre system may be recommended to produce 'Cabernet Franc' grapes regarding soil and climatic conditions of the Serra Gaúcha region.
\end{abstract}

Index terms: Viticulture, grape, must, chemical composition.

(Recebido para publicação em 22 de setembro de 2004 e aprovado em 15 de março de 2006)

A cultura da videira na Serra Gaúcha ocupa um lugar de destaque na fruticultura do Rio Grande do Sul. Visando a melhorar a funcionalidade e o rendimento econômico dos vinhedos e a qualidade da uva, sistemas alternativos de condução vêm sendo utilizados pelos viticultores da região, entre eles o sistema lira aberta.

A videira é uma das poucas espécies de fruteiras que necessitam de sustentação. A condução se refere ao método que define a forma da planta, ou seja, a arquitetura do vinhedo Winkler (1969). A condução da videira visa à domesticá-la através do emprego de um certo número de práticas culturais coordenadas, concorrendo para o mesmo objetivo (BRANAS, 1965). Esses objetivos são produzir plantas com um formato que facilite seu cultivo, o controle de doenças e pragas, a poda e a colheita da uva (WINKLER, 1969).

A qualidade do fruto depende em grande parte da radiação solar recebida durante o período de crescimento vegetativo (INTRIERI \& FILIPPETTI, 2000). A exposição direta da uva à radiação solar causa aumento do tamanho da baga e acumulação de açúcar na uva. Como conseqüência, ocorre a redução da acidez total pela degradação do ácido málico, aumento da concentração de antocianinas e diminuição do desenvolvimento de podridões (RIBÉREAU-GAYON et al., 1976; SMART \& ROBINSON, 1994). Por outro lado, o sombreamento do fruto interfere em sua composição, reduzindo a concentração de açúcar, a relação glicose/frutose, os polifenóis e as antocianinas e aumentando as concentrações de N, Mg, Ca, K e ácido málico (SMART et al., 1988). Além disso, o sistema de condução em lira proporciona uma melhora na maturação da uva, qualidade do vinho $(\mathrm{pH}$, açúcares, notas aromáticas, quantidade e qualidade de polifenóis), regularidade de produção e redução dos custos de produção (CARBONNEAU, 1980).

Em função do que foi exposto acima, objetivou-se com este trabalho avaliar a composição físico-química do

${ }^{1}$ Engenheiro Agrônomo, Dr. - Centro Federal de Educação Tecnológica/CEFET - Petrolina - Br 235, Km 22 - PSNC N4 - Cx. P. 178 - 56.302-970 Petrolina, PE - Imanfroi@bol.com.br

Engenheiro Agrônomo, Dr., Embrapa Uva e Vinho - Cx. P. 130 - 95.700-000 - Bento Gonçalves, RS.

${ }^{3}$ Engenheiro Agrônomo, Dr. - Universidade Federal do Rio Grande do Sul/UFRGS - Departamento de Horticultura e Silvicultura - Cx. P. 776 91.501-970 - Porto Alegre, RS. 
mosto da cv. Cabernet Franc conduzida no sistema lira aberta na Serra Gaúcha.

O trabalho foi realizado no ciclo vegetativo de 1995/ 1996 em oito vinhedos de 'Cabernet Franc', localizados em diferentes áreas dos municípios de Bento Gonçalves (comunidades de São Valentim, Tuiuti e Pinto Bandeira) e Monte Belo do Sul, Rio Grande do Sul. Os vinhedos foram instalados em 1989 e conduzidos no sistema lira aberta. As mudas da cv. Cabernet Franc foram importadas da França e enxertadas sobre o porta-enxerto SO4.

O sistema lira aberta caracterizou-se pela condução das videiras em dois planos verticais oblíquos, mantidos por três fios em cada um dos planos. O espaçamento de plantio variou de 1,00 a 1,35 m entre plantas e de 2,50 a 3,10 $\mathrm{m}$ entre fileiras. A poda seca foi a mista, deixando-se, em média, 4,3 varas/planta com 5,3 gemas/vara e 11,9 esporões/ planta com 1,3 gema/esporão, ou seja, 38,3 gemas/planta.

Por ocasião da maturação da uva, que ocorreu em fevereiro, colheram-se aproximadamente $2 \mathrm{~kg}$ de uva/planta, totalizando $40 \mathrm{~kg}$ por vinhedo, ou seja, $20 \mathrm{~kg}$ para cada repetição. Nos vinhedos que foram atingidos pela podridão cinzenta da uva a colheita foi antecipada em média quatro dias. Esta uva foi posteriormente desengaçada e esmagada para a obtenção do mosto no Laboratório de Microvinificação da Embrapa Uva e Vinho.

As análises do mosto foram realizadas no Laboratório de Enoquímica dessa instituição. O teor de sólidos solúveis totais (SST), expresso em ${ }^{\circ}$ Brix, foi determinado através de um refratômetro digital de bancada com controle automático de temperatura; a densidade, através de um densímetro digital marca Anton Paar, modelo DMA-45; a acidez total, expressa em ácido tartárico, pela titulação do mosto com $\mathrm{NaOH} 0,1 \mathrm{~N}$, utilizando o azul de bromotimol como indicador; o $\mathrm{pH}$, com um potenciômetro digital marca Corning, modelo 125, equipado com eletrodo de vidro e calibrado com solução padrão de pH 3,0 e pH 4,0.

Os polifenóis totais foram determinados medindose a absorbância através de espectrofotômetro UV/VIS a 280 nm (RIBÉREAU-GAYON et al., 1986); a prolina e o nitrogênio amoniacal e total, segundo metodologia proposta por Giannessi \& Matta (1987); os açúcares redutores, segundo Meyer \& Leygue-Alba (1991).

Os ácidos orgânicos foram analisados através da cromatografia líquida de alta performance, segundo método descrito por Auguste (1979); para a determinação dos minerais foi utilizada a metodologia proposta por PerkinElmer (2000), através de um espectrofotômetro de absorção atômica Perkin-Elmer, modelo 2380, com lâmpadas de cátodo oco para cada elemento. O Ca, Mg, Mn, Zn, Cu e Fe foram determinados por absorção atômica e o K, Na, Li e Rb, por emissão de chama; para a análise do $\mathrm{P}$ utilizou-se a metodologia proposta por Tedesco et al. (1995), adaptada para mostos e vinhos.

Os valores do ${ }^{\circ}$ Brix, densidade e açúcares redutores encontram-se na Tabela 1 . A média do ${ }^{\circ}$ Brix foi de 17,0 , sendo que os menores valores foram observados em vinhedos onde a colheita foi antecipada. Os baixos valores de ${ }^{\circ}$ Brix foram também influenciados pelo excesso de vigor das plantas, adubação nitrogenada dos vinhedos e pela precipitação pluviométrica que ocorreu entre a mudança de cor e a colheita.

Em concordância com estes resultados, estão os trabalhos desenvolvidos por outros autores com a cv. Cabernet Franc conduzida em latada (MIELE et al., 1982; PASSOS \& TRINTIN, 1982; RIZZON et al., 1986). No que diz respeito ao manejo, vinhedos com altos índices de área foliar proporcionam maior sombreamento e, por isso, reduzem a concentração de açúcar da uva (SMART et al., 1985). Por outro lado, boa exposição das bagas aos raios solares faz com que a concentração de açúcares aumente (CRIPPEN JUNIOR \& MORRISON, 1986).

A densidade média do mosto encontrada no presente trabalho foi de $1,0728 \mathrm{~g} \mathrm{~mL}^{-1}$, ou seja, a concentração de açúcares é a variável que mais influencia na densidade do mosto. A concentração média dos açúcares redutores foi de $162,9 \mathrm{~g} \mathrm{~L}^{-1}$, denotando a maior capacidade que as cultivares européias têm de produzir açúcar em relação às americanas mais tradicionais cultivadas na região.

O valor médio de acidez total (Tabela 1) foi de 113 meq $\mathrm{L}^{-1}$, considerado adequado para elaboração de vinho tinto. Esta acidez é resultante, principalmente, da concentração dos ácidos tartárico e málico presentes na baga da uva. Normalmente, estes valores diminuem bastante durante a elaboração do vinho.

$\mathrm{O}$ valor médio da relação ${ }^{\circ}$ Brix/Acidez total (Tabela 1 ) foi de 20,3, com valores mínimos e máximos muito distanciados (15,9 e 29,5, respectivamente). Esta variação deveu-se mais à concentração dos ácidos do que à dos açúcares presentes na uva.

$\mathrm{O}$ pH variou de 3,05 a 3,31 (média de 3,17) (Tabela 1). $\mathrm{O}$ pH possui uma grande relação com as concentrações de ácido tartárico da uva e de minerais do solo, principalmente do $\mathrm{K}$, adubação nitrogenada e também da precipitação pluviométrica. Segundo Aerny (1985), mostos com pH baixo estão mais protegidos da ação de enzimas oxidativas. Resultados similares foram encontrados por Rizzon et al. (1998) no estudo da evolução da acidez durante a vinificação de uvas tintas. 


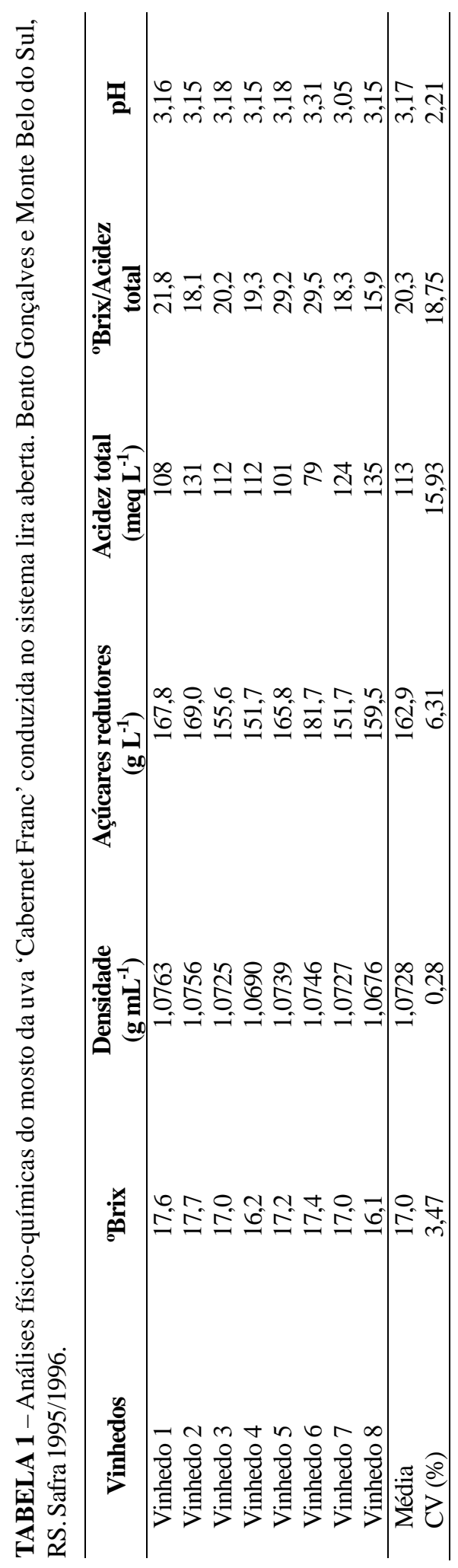

Os resultados do nitrogênio total e do nitrogênio amoniacal do mosto apresentaram uma grande variação (Tabela 2). O nitrogênio total variou de $157,5 \mathrm{mg} \mathrm{L}^{-1}$ a 332,5 $\mathrm{mg} \mathrm{L}^{-1}$ e o nitrogênio amoniacal de $16,6 \mathrm{mg} \mathrm{L}^{-1}$ a $70,9 \mathrm{mg} \mathrm{L}^{-1}$, com médias de $246,1 \mathrm{mg} \mathrm{L}^{-1}$ e $43,6 \mathrm{mg} \mathrm{L}^{-1}$, respectivamente. As concentrações baixas podem ter sido resultado da presença de taninos que reagiriam com as proteínas e as precipitariam (RIZZON \& MIELE, 2001). Segundo Amerine \& Ough (1976), a concentração de nitrogênio encontrada no mosto pode variar de $24 \mathrm{mg} \mathrm{L}^{-1}$ a $309 \mathrm{mg} \mathrm{L}^{-1}$, dependendo da variedade.

Os polifenóis totais (Tabela 2) variaram de 10,9 a 13,9. Este grupo de compostos encontra-se na película e a sua concentração está relacionada com a maturação da uva. Como no presente trabalho a uva sofreu somente o desengace e um leve esmagamento, os valores de polífenóis totais foram baixos.

A concentração média de prolina foi baixa (182,2 $\left.\mathrm{mg} \mathrm{L}^{-1}\right)$, ocorrendo grande variação entre os vinhedos, com mínimo de 21,3 $\mathrm{mg} \mathrm{L}^{-1}$ e máximo de 497,9 $\mathrm{mg} \mathrm{L}^{-1}$ (Tabela 2). Isto ocorreu devido, principalmente, ao grau de maturação e de sanidade da uva. Segundo Bisson (1991), o teor de prolina é uma característica genética da videira, sendo fortemente influenciada pelas condições climáticas da região, adubação nitrogenada e estado de sanidade da uva.

A concentração dos ácidos tartárico e málico varia de acordo com o vinhedo, clima, ano e estado de maturação da uva (RIBÉREAU-GAYON et al., 1986). O teor do ácido tartárico foi de $88 \mathrm{meq} \mathrm{L}^{-1}$ e o do ácido málico, de $56 \mathrm{meq} \mathrm{L}^{-1}$ (Tabela 2). A soma dos dois ácidos foi de $144 \mathrm{meq} \mathrm{L}^{-1} \mathrm{e}$ a relação ácido tartárico/ácido málico foi de 1,71.

A concentração média dos minerais do mosto está relacionada na Tabela 3. O K é o cátion que predomina no mosto, tornando-se mais solúvel à medida que ocorre a maturação da uva. Neste caso, a concentração foi de $951 \mathrm{mg} \mathrm{L}^{-1}$, menor que a encontrada por Rizzon et al. (1986), de $1.250 \mathrm{mg} \mathrm{L}^{-1}$; o P, importante para determinadas fases da fermentação, foi o segundo mineral em concentração (71,3 $\mathrm{mg} \mathrm{L}^{-1}$ ), podendo chegar a $150 \mathrm{mg} \mathrm{L}^{-1}$ (WEBB, 1974); a de $\mathrm{Mg}$, encontrado em maior quantidade em vinhos tintos, foi de 50,2 $\mathrm{mg} \mathrm{L}^{-1}$; a de Ca foi relativamente baixa (34,4 $\left.\mathrm{mg} \mathrm{L}^{-1}\right)$, pois, normalmente, os solos da região apresentam baixa concentração deste mineral; a de Na também foi baixa (3,1 $\left.\mathrm{mg} \mathrm{L}^{-1}\right)$, como conseqüência das condições de alta umidade da região produtora de uva; e a do $\mathrm{Cu}\left(5,4 \mathrm{mg} \mathrm{L}^{-1}\right)$ apresentou valores demasiadamente altos para o mosto, o que é explicado pela quantidade e época de aplicação da calda bordalesa pulverizada como fungicida. 

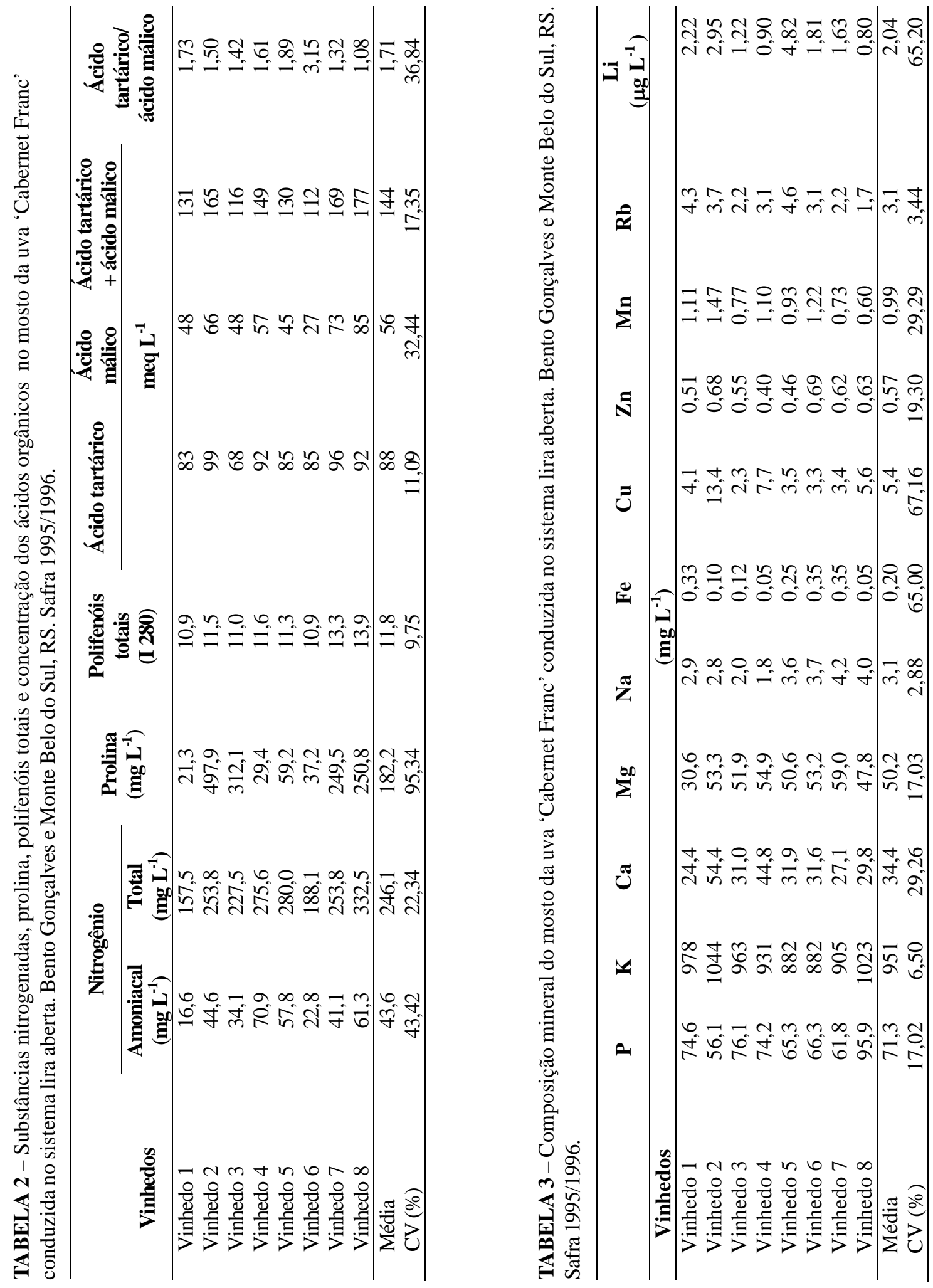

Ciênc. agrotec., Lavras, v. 30, n. 4, p. 787-792, jul./ago., 2006 
Os demais minerais também apresentaram concentrações normais às encontradas em mostos da região: Fe 0,20 $\mathrm{mg} \mathrm{L}^{-1}$, Zn 0,57 mg L ${ }^{-1}$, Mn 0,99 $\mathrm{mg} \mathrm{L}^{-1}$, Rb 3,10 $\mathrm{mg} \mathrm{L}^{-1} \mathrm{e} \mathrm{Li}$ 2,04 $\mathrm{mg} \mathrm{L}^{-1}$.

Sumarizando, as análises físico-químicas do mosto da uva 'Cabernet Franc' conduzida no sistema lira aberta na Serra Gaúcha, caracterizaram-se por apresentar concentrações de açúcar, ácidos orgânicos e nitrogênio total normais para as condições da região, $\mathrm{pH}$ baixo e concentrações relativamente baixas de prolina e de cobre em alguns vinhedos. Para as demais variáveis, os resultados foram similares aos verificados em mostos provenientes de videiras conduzidas em latada e espaldeira nesta região em anos anteriores.

\section{CONCLUSÃO}

O sistema de condução da videira em lira pode ser recomendado para a produção de uva 'Cabernet Franc' destinada à elaboração de vinho fino, considerando as condições de solo e de clima da Serra Gaúcha.

\section{REFERÊNCIAS BIBLIOGRÁFICAS}

AERNY, J. Définition de la qualité de la vendange. Revue Suisse Viticulture, Arboriculture, Horticulture, Nyon, v. 17 , n. 4 , p. $219-223,1985$.

AMERINE, M. A.; OUGH, C. S. Análisis de vinos y mostos. Zaragoza: Acribia, 1976. 158 p.

AUGUSTE, M. H. Application de la chromatographie en phase liquide à haute pression à l'analyse des moûts et des vins. 1979. 135 f. Tese (Doctorat en OenologieAmpélologie) - Institut d'Oenologie, Université de Bordeaux II, Talence, 1979.

BISSON, J. F. Influence of nitrogen on yeast and fermentation of grapes. In: INTERNATIONAL SYMPOSIUM ON NITROGEN IN GRAPES AND WINE, 1., 1991, Seattle, WA. Proceedings... Seattle: The American Society for Enology and Viticulture, 1991. p. 78-89.

BRANAS, J. Bases générales de la taille de la vigne. Le Progrès Agricole et Viticole, Montpellier, v. 82, n. 22, p. 311-318, 1965.

CARBONNEAU, A. Recherche sur les systèmes de conduite de la vigne: essai de maitrise du microclimat et de la plante entière pour produire économiquement du raisin de qualité. 1980. 235 f. Tese (Doctotrat en Oenologie-
Ampélologie) - Institut d'Oenologie, Université de Bordeaux II, Talence, 1980.

CRIPPEN JUNIOR, D. D.; MORRISON, J. C. The effects of sun exposure on the compositional development of Cabernet Sauvignon berries. American Journal of Enology and Viticulture, Davis, v. 37, n. 4, p. 235-242, 1986.

GIANNESSI, P.; MATTA, M. Trattato di scienza e tecnica enologica: analisi e controllo dei mosti e dei vini. Brescia: AEB, 1987. v. 1, 349 p.

INTRIERI, C.; FILIPPETTI, I. Innovations and outlook in grapevine training systems and mechanization in NorthCentral Italy. In: AMERICAN SOCIETY FOR ENOLOGY AND VITICULTURE ANNUAL MEETING, 50., 2000, Seattle, WA. Proceedings... Seattle: The American Society for Enology and Viticulture, 2000. p. 170-184.

MEYER, C. R.; LEYGUE-ALBA, N. M. R. Manual de métodos analíticos enológicos. Caxias do Sul: UCS, 1991. $51 \mathrm{p}$.

MIELE, A.; IGNACZAK, J. C.; PEREIRA, F. M. Efeito da calciocianamida na quebra de dormência, fertilidade das gemas, produtividade do vinhedo e qualidade da uva Cabernet Franc. Pesquisa Agropecuária Brasileira, Brasília, v. 17, n. 3, p. 393-398, 1982.

PASSOS, L. P.; TRINTIN, P. L. Resposta da videira à variação da época de poda seca: V. efeitos na qualidade do fruto da cv. Cabernet Franc. Bento Gonçalves: EmbrapaCNPUV, 1982. 4 p. (Pesquisa em Andamento, 8).

PERKIN-ELMER. Analytical methods for atomic absorption spectrophotometry. Singapura: Perkin-Elmer, 2000. 300 p.

RIBÉREAU-GAYON, J.; PEYNAUD, E.; SUDRAUD, P.; RIBÉREAU-GAYON, P. Sciences et techniques du vin. Paris: Dunod, 1976. v. 1, 671 p.

RIBÉREAU-GAYON, J.; PEYNAUD, E.; SUDRAUD, P.; RIBÉREAU-GAYON, P. Carattere dei vini, maturazione dell'uva, lieviti e batteri: trattato di scienza e tecnica enologica. Brescia: AEB, 1986. v. 2, 424 p.

RIZZON, L. A.; MIELE, A. Avaliação da cv. Cabernet Franc para elaboração de vinho tinto. Ciência e Tecnologia de Alimentos, Campinas, v. 21, n. 2, p. 249-255, 2001. 
RIZZON, L. A.; MIELE, A.; MENEGUZZO, J. Avaliação enológica de algumas cultivares de videira. Bento Gonçalves: Embrapa-CNPUV, 1986. 11 p. (PNP Vitivinicultura. Projeto 020.86.001/1).

RIZZON, L. A.; ZANUZ, M. C.; MIELE, A. Evolução da acidez durante a vinificação de uvas tintas de três regiões vitícolas do Rio Grande do Sul. Ciência e Tecnologia de Alimentos, Campinas, v. 18, n. 2, p. 179-183, 1998.

SMART, R. E.; ROBINSON, M. D. Sunlight into wine: a handbook for winegrape canopy management. 3. ed. Adelaide: Winetitles, 1994. 88 p.

SMART, R. E.; ROBINSON, J. B.; DUE, G. R.; BRIEN, C. J. Canopy microclimate modification for the cultivar Shiraz: II. effects on must and wine composition. Vitis, Siebeldingen, v. 24, n. 2, p. 119-128, 1985.
SMART, R. E.; SMITH, S. M.; WINCHESTER, R. V. Light quality and quantity effects on fruit ripening for Cabernet Sauvignon. American Journal of Enology and Viticulture, Davis, v. 39, n. 3, p. 250258, 1988.

TEDESCO, M. J.; GIANELLO, C.; BISSANI, C. A.; BOHNEN, H.; VOLKWEISS, S. J. Análise de solo, plantas e outros materiais. 2. ed. Porto Alegre: UFRGS, 1995. 174 p. (Boletim Técnico, 5).

WEBB, A. D. Chemistry of winemaking. Washington: American Chemical Society, 1974. 311 p.

WINKLER, A. J. Effect of vine spacing in an unirrigated vineyard on vine physiology, production and wine quality. American Journal of Enology and Viticulture, Davis, v. 20, p. 7-15, 1969.

Ciênc. agrotec., Lavras, v. 30, n. 4, p. 787-792, jul./ago., 2006 